\title{
Urgent double balloon endoscopy provides higher yields than non-urgent double balloon endoscopy in overt obscure gastrointestinal bleeding
}

Authors

Institution
Satimai Aniwan, Vichai Viriyautsahakul, Rungsun Rerknimitr, Phonthep Angsuwatcharakon, Pradermchai Kongkam, Sombat Treeprasertsuk, Pinit Kullavanijaya

Division of Gastroenterology, Department of Medicine, Faculty of Medicine, Chulalongkorn University, Bangkok, Thailand submitted 18. October 2013 accepted after revision 23. January 2014

Bibliography Dol http://dx.doi.org/ 10.1055/s-0034-1365543 Published online: 7.5.2014 Endoscopy International Open 2014; 02: E90-E95

(c) Georg Thieme Verlag KG Stuttgart · New York E-ISSN 2196-9736

Corresponding author Rungsun Rerknimitr, MD Division of Gastroenterology, Department of Medicine, Faculty of Medicine. Chulalongkorn University Bangkok 10310

Thailand

ERCP@live.com
Background and study aims: In overt obscure gastrointestinal bleeding (OV), double balloon endoscopy (DBE) is recommended as one of the most important investigations as it can provide both diagnosis and treatment. However, there is no set standard on the timing of DBE in OV. The aim of this study was to compare the diagnostic and therapeutic yields between urgent and nonurgent DBE in patients with OV.

Patients and methods: Between January 2006 and February 2013, 120 patients with OV who underwent DBE were retrospectively reviewed. An urgent DBE was defined as DBE performed within $72 \mathrm{~h}$ from the last visible gastrointestinal bleeding $(\mathrm{n}=74)$ whereas a non-urgent DBE was defined as DBE performed after $72 \mathrm{~h}(\mathrm{n}=46)$. Diagnostic yields, therapeutic impact and clinical outcomes were evaluated.

\section{Introduction}

Traditionally, obscure gastrointestinal bleeding can be classified into overt-obscure bleeding $(\mathrm{OV})$ and occult-obscure bleeding (OC). OV is defined as visible gastrointestinal bleeding of unknown origin that either recurs or does not recur after an initial negative colonoscopy and gastroscopy. OC, however, is defined as GI blood loss without visible bleeding [1]. Technically, OV requires prompt diagnosis and treatment [2-4]. Approximately, $75 \%$ of OV sources are located in the small intestine [5]. One of the most important investigations in OV is double balloon endoscopy (DBE) as it can provide not only total small bowel examination but also endoscopic therapy. It has been confirmed that if bleeding sources can be identified, recurrent bleeding would be low [6]. Diagnostic yields of DBE in obscure gastrointestinal bleeding have been reported to vary between $41 \%$ and $80 \%$ [6-8]. One of the important factors resulting in a wide range of diagnostic yields may
Results: Diagnostic yield in urgent DBE was significantly higher than that in non-urgent DBE (70\% versus $30 \%$; $P<0.05$ ). Urgent DBE offered significantly more therapies including endoscopic, angiographic embolization, and surgery than nonurgent DBE (54\% versus $15 \%$; $P<0.001$ ). Endoscopic therapy was performed in $43 \%$ of urgentDBE patients whereas only $13 \%$ of patients in the other group received endoscopic therapy $(P<$ 0.01 ). In patients with identified bleeding sources, the rebleeding rate was lower in patients who underwent urgent DBE than in those who underwent non-urgent DBE (10\% versus $29 \%$, NS).

Conclusions: Regarding diagnostic and therapeutic impacts in OV, our retrospective study showed that urgent DBE is better than non-urgent DBE. The recurrent bleeding rate in patients undergoing urgent DBE tended to be lower.

be the differences in the proportion of OV and OC in different series. In addition, the timing of DBE plays an important role for the different outcomes $[2-4,9]$. Hypothetically, vascular lesions and/or ulcers may be difficult to identify if the investigation is delayed [9].

Many series have supported the view that early investigation in active or overt bleeding could result in a higher diagnostic yield. Patients with OV and $\mathrm{OC}$ who underwent capsule endoscopy had a diagnostic yield of $90 \%$ and $62 \%$, respectively [10]. Another recent retrospective review study also confirmed that an emergency capsule endoscopy provided a high diagnostic yield (67\%) in patients with OV [3]. Additionally, in a subgroup of OV, patients with ongoing OV had more benefit from capsule endoscopy than OV patients without ongoing bleeding (92\% versus 13\%) [10]. However, there was only one report of emergency DBE in only 10 cases of OV from Germany giving the highest diagnostic yield (90\%) [2]. 
DBE can provide both diagnosis and therapy at the same time but capsule endoscopy needs at least $8-12 \mathrm{~h}$ for a complete examination but without therapy. Because of this advantage, DBE may be considered to be the first choice in an OV, especially in a patient with ongoing bleeding [9]. However, there is no set standard on the optimum timing of DBE in OV.

Therefore, the objective of this study was to compare an urgent DBE with a non-urgent DBE with the following aims: 1) to evaluate the diagnostic yield, 2) to evaluate the therapeutic impact, and 3 ) to analyze the impact on rebleeding rate and clinical outcomes of patients with OV.

\section{Patients and methods \\ $\nabla$}

\section{Definition}

In this study, OV was defined as visible gastrointestinal bleeding of unknown origin that developed with or without recurrence after an initial negative esophagogastroduodenoscopy (EGD) and colonoscopy [5]. An urgent DBE was defined as DBE performed within $72 \mathrm{~h}$ from the last visible gastrointestinal bleeding whereas non-urgent DBE was defined as DBE performed after 72 h. We classified patients with OV into three types: 1) Ongoing OV patient was a patient with evidence of bleeding such as melena or hematochezia still present on the day of DBE; 2) Recent OV patient was a patient with evidence of bleeding within $72 \mathrm{~h}$ but no ongoing OV on the day of DBE; 3) Previous OV patient was a patient with evidence of bleeding prior to $72 \mathrm{~h}$ before the DBE.

\section{Patients}

Of the 261 patients who underwent DBE between January 2006 and February 2013, 120 patients with OV were retrospectively reviewed. The other 141 patients underwent DBE for non-OV indications (chronic diarrhea $n=55$, occult obscure gastrointestinal bleeding $n=38$, small bowel obstruction $n=14$, Peutz-Jeghers syndrome $n=12$, chronic abdominal pain $n=8$, Crohn's disease $n=7$, suspected small bowel tumors $n=7$ ) so were excluded. Clinical characteristics, endoscopic procedures, and therapeutic interventions were reviewed. Clinical outcomes such as rebleeding rate, subsequent therapeutic requirement, amount of blood transfused, and length of hospital stay were reviewed. Follow-up data were obtained from patient medical records and phone contacts. All patients underwent EGD and colonoscopy prior to DBE. During the study period, capsule endoscopy was not available in our institution. This study was approved by the Institutional Review Board of the Faculty of Medicine, Chulalongkorn University.

\section{DBE procedure}

A standard DBE system (EN-450P5/28, Fujifilm, Tokyo, Japan) was used for small intestine examination. The procedure was performed using the DBE technique described by Yamamoto et al. [7]. In our practice, we performed DBE as early as possible in all patients with $\mathrm{OV}$. However, some patients were referred from other hospitals and needed repeat EGD and colonoscopy. Also, in many cases, the bleeding ceased spontaneously before reaching our hospital, so in some patients, DBE was not possible before $72 \mathrm{~h}$. The oral approach was chosen first in patients with melena, whereas the anal approach was chosen for patients with hematochezia. If the first approach was negative, the other route was used. When bidirectional approaches were necessary, sterilized India ink was tattooed as a landmark at the furthest depth of the initial route. Patients took 2-4L of polyethylene glycol as an additional bowel preparation only when the effect of a recent bowel preparation for colonoscopy was deemed as inadequate. After obtaining written informed consent, DBE was performed in all patients under a conscious sedation with midazolam and/or meperidine administration and a standard cardiorespiratory monitoring was provided to all.

\section{Definition of the bleeding source}

We categorized the sources of bleeding as ulcers (more than 10 $\mathrm{mm}$ in diameter), vascular lesions according to the classification by Yano et al. [11], tumors/polyps with ulcer/erosion, and diverticula with ulcers/vessels described elsewhere [12,13]. In addition, all small bowel tumors larger than $2 \mathrm{~cm}$ with or without ulceration were considered to be bleeding sources. We did not consider the following to be bleeding sources: angiodysplasia (less than 1 $\mathrm{mm}$ without oozing), non-bleeding polyp, small lipoma, lymphangiectasia, diverticula of the small bowel without ulcers/vessels $[12,13]$.

\section{Statistical analysis}

The proportion of patients with positive findings from urgent and non-urgent DBE was compared using the Chi-square test. Data were presented as the mean, median and range. $P$-values less than 0.05 were considered to be statistically significant. The statistical analyses were performed using SPSS version 17.0 (SPSS Inc., Chicago, IL).

\section{Results \\ $\nabla$}

One-hundred and twenty patients with OV who underwent DBE at our gastrointestinal endoscopy unit were included in this study. Of the 120 patients, 24 patients (20\%) with ongoing OV and 50 patients (42\%) with recent OV all underwent urgent DBE $(\mathrm{n}=74)$, whereas the other 46 patients $(38 \%)$ were categorized as previous OV and underwent non-urgent DBE ( $\bullet$ Fig.1). Mean

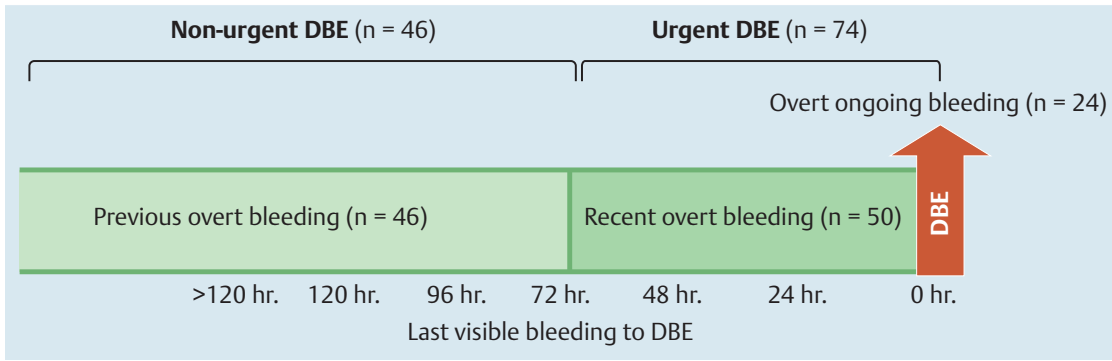

Fig. 1 Classification of overt obscure bleeding based on time of last visible bleeding to time of DBE. 
Table 1 Baseline characteristics of the patients.

\begin{tabular}{|c|c|c|c|}
\hline Baseline characteristics & $\begin{array}{l}\text { Urgent DBE } \\
(n=74)\end{array}$ & $\begin{array}{l}\text { Non-urgent } \\
\text { DBE }(n=46)\end{array}$ & $P$-value \\
\hline Mean age (years) & $60 \pm 2.4$ & $56 \pm 2.9$ & 0.37 \\
\hline Female & $42(57 \%)$ & $21(46 \%)$ & 0.19 \\
\hline $\begin{array}{l}\text { Mean initial hemoglobin } \\
(\mathrm{g} / \mathrm{dl})\end{array}$ & $7.1 \pm 0.2$ & $7.8 \pm 0.3$ & 0.13 \\
\hline $\begin{array}{l}\text { Median units of blood } \\
\text { transfused before DBE }\end{array}$ & $4(3-7)$ & $2(1-4.3)$ & 0.005 \\
\hline $\begin{array}{l}\text { Median time from last } \\
\text { visible bleeding to DBE } \\
\text { (h, range) }\end{array}$ & $48(0-72)$ & $168(84-720)$ & $<0.001$ \\
\hline
\end{tabular}

age, gender, and hemoglobin level at presentation were similar between the two groups. Patients in the urgent DBE group received an average of 4 units of blood transfused before going to DBE and this amount was more than that in the non-urgent DBE group (2 units). DBE was performed within a median time of 48 $\mathrm{h}$ (range $0-72 \mathrm{~h}$ ) after the last visible bleeding in the urgent DBE group and within a median time of $168 \mathrm{~h}$ (range $84-720 \mathrm{~h}$ ) in the non-urgent DBE group. Apart from the amount of blood transfused and the intervening time before DBE, all other baseline characteristics between the two groups were not statistically significantly different ( $\bullet$ Table 1 ). DBE was performed using an oral approach only, anal approach only, and combined approach in 65 (54\%), $30(25 \%)$, and $25(21 \%)$ patients, respectively. The success rate of total small bowel examination was $68 \%$ in the 25 attempts.

\section{Diagnostic yields}

The overall diagnostic yield of DBE in OV was significantly higher in the urgent DBE group than in the non-urgent DBE group (70\% versus $30 \%, P<0.001$ ). Active bleeding (spurting or oozing) was found in 20 (27\%) patients with urgent DBE whereas there were only 3 (7\%) identifiable active bleedings in the non-urgent DBE group $(P<0.01)$. Bleeding sources in each group are shown in - Table2. Ulcers were the most common bleeding sources; $27 \%$

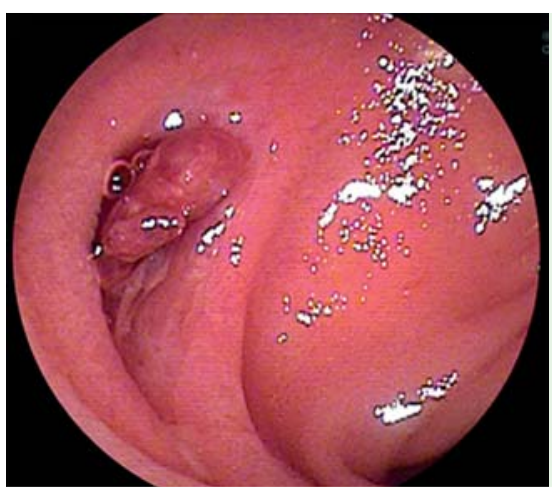

Fig. 2 Jejunal ulcer with visible vessel detected during an urgent DBE.

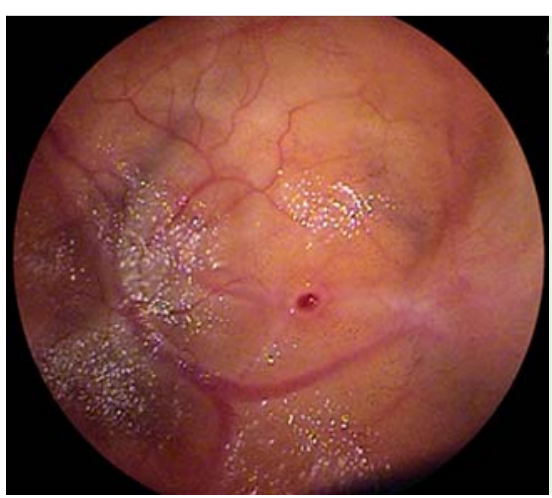

Fig. 3 Bleeding diverticulum detected during an urgent DBE.

in the urgent group ( $\bullet$ Fig. 2 ) and $17 \%$ in the non-urgent group $(P>0.05)$. Vascular lesions were diagnosed more frequently in the urgent group (13 angiodysplasia, 5 small bowel varices) than in the non-urgent group ( 3 angiodysplasia, 1 small bowel varices) ( $24 \%$ versus $9 \% ; P=0.05$, respectively). Three patients with jejunal diverticular bleeding ( $\bullet$ Fig. 3 ) and 2 patients with Meckel's diverticular bleeding were detected only in the urgent group. Among 5 patients with small bowel tumor bleeding, 4 patients were from the urgent group and one was from the non-urgent group.

\begin{tabular}{|c|c|c|c|}
\hline & $\begin{array}{l}\text { Urgent DBE } \\
(n=74)\end{array}$ & Non-urgent DBE $(n=46)$ & $P$-value \\
\hline Etiology of OV & $52(70 \%)$ & $14(30 \%)$ & $<0.001$ \\
\hline Ulcer & $20(27 \%)$ & $8(17 \%)$ & \\
\hline Inactive bleeding angiodysplasia & $9(12 \%)$ & $3(7 \%)$ & \\
\hline Active bleeding angiodysplasia & $4(5 \%)$ & $0(0 \%)$ & \\
\hline Small bowel varices & $5(7 \%)$ & $1(2 \%)$ & \\
\hline Small bowel tumor & $4(5 \%)$ & $1(2 \%)$ & \\
\hline Bleeding diverticulum & $3(4 \%)$ & $0(0 \%)$ & \\
\hline Bleeding polyp & $2(3 \%)$ & $1(2 \%)$ & \\
\hline Portal hypertensive enteropathy & $2(3 \%)$ & $0(0 \%)$ & \\
\hline Meckel's diverticulum & $2(3 \%)$ & $0(0 \%)$ & \\
\hline Hemobilia & $1(1 \%)$ & $0(0 \%)$ & \\
\hline $\begin{array}{l}\text { Modification in management by DBE } \\
\text { results }\end{array}$ & $40(54 \%)$ & $7(15 \%)$ & $<0.001$ \\
\hline Endoscopic therapies & $32(43 \%)$ & $6(13 \%)$ & 0.001 \\
\hline Argon plasma coagulation & $17(23 \%)$ & $4(9 \%)$ & \\
\hline Adrenaline injection & $4(5 \%)$ & $1(2 \%)$ & \\
\hline Glue injection & $4(5 \%)$ & $0(0 \%)$ & \\
\hline Clipping & $4(5 \%)$ & $0(0 \%)$ & \\
\hline Bipolar coaptation & $2(3 \%)$ & $0(0 \%)$ & \\
\hline Polypectomy & $1(1 \%)$ & $1(2 \%)$ & \\
\hline Surgery & $9(12 \%)$ & $1(2 \%)$ & 0.16 \\
\hline
\end{tabular}

Table 2 Bleeding etiologies and therapeutic impact in patients with urgent and non-urgent DBE. 
Subgroup analysis of the diagnostic yields in ongoing and recent OV patients found the diagnostic yields to be $75 \%$ (18/24 patients) and $68 \%$ (34/50 patients), respectively $(P=0.67)$.

\section{Therapeutic impact ( 0 Table 2)}

Therapies including endoscopic, angiographic embolization, and surgery for overt OGIB were performed in 40 (54\%) patients who underwent urgent DBE, whereas only $7(15 \%)$ patients in the non-urgent group received therapies $(P<0.001)$. Among patients who underwent urgent DBE, endoscopic therapy was performed in $32(43 \%)$ patients but only $6(13 \%)$ patients in the non-urgent group received endoscopic therapy $(P<0.01)$. Nine $(12 \%)$ patients in the urgent group and $1(2 \%)$ in the non-urgent group underwent surgery. Two patients in the urgent group with negative DBE proceeded to angiography, but bleeding sources were not identified. Consequently, 1 patient died from severe sepsis 4 months later, whereas the other did not have recurrent bleeding during the 6-month follow-up.

\section{Clinical outcomes}

Among the 120 patients, follow-up data were available in 97 (80\%) patients including 60 (out of 74 , or $81 \%$ ) patients in the urgent group and 37 (out of 46 , or $80 \%$ ) patients in the non-urgent group. The mean follow-up period was $16.3 \pm 1.8$ months. Of those 66 patients with bleeding sources identified by DBE, the urgent group had a lower rebleeding rate than the non-urgent group (5 of 52 patients, $10 \%$ versus 4 of 14 patients, $29 \%$, respectively) but this was not statistically significant $(P=0.08)$. In the urgent group, 3 of 5 underwent repeat DBE. One patient was diagnosed with multiple angiodysplasia and the other 2 were diagnosed with small bowel varices and all 3 received endoscopic therapy. Two patients with ulcers and diverticular bleeding diagnosed during the first DBE received conservative treatment successfully. In the non-urgent group, 3 of 4 patients previously diagnosed with multiple ulcers underwent repeat DBE. One patient required a second endoscopic therapy whereas the other 2 were managed conservatively. To date, none have developed recurrent bleeding. The mean blood transfusion requirement after DBE and the length of hospital stay between the two groups were not statistically different ( Table 3). No patient developed any serious complication and no GI bleeding-related death occurred during the 18-month follow-up.

\section{Discussion}

Recent studies have proposed that patients with OV could be categorized into two subgroups (ongoing OV and previous OV) [9, 14]. A patient seen with active bleeding is classified as ongoing $\mathrm{OV}$, a patient without ongoing bleeding but with a previous epi-

Table 3 Clinical outcomes in 66 patients with identifiable bleeding source.

\begin{tabular}{|lcc|}
\hline & $\begin{array}{l}\text { Urgent DBE } \\
(\mathbf{n = 5 2 )}\end{array}$ & $\begin{array}{c}\text { Non-urgent } \\
\text { DBE (n=14) }\end{array}$ \\
\hline Rebleeding & $5(10 \%)$ & $4(29 \%)$ \\
\hline $\begin{array}{l}\text { Hospital stay (days, median + IQR) } \\
\text { meod transfused after DBE (units, }\end{array}$ & $12(7-38)$ & $15(10-22)$ \\
\hline
\end{tabular}

Abbreviation: IQR, interquartile range. $P>0.05$. sode of overt bleeding is classified as previous OV [9]. Earlier studies demonstrated significant differences in diagnostic yield of DBE between these two bleeding types $[9,14]$. The diagnostic yields of DBE in patients with ongoing OV and previous OV were reportedly different (77-100\% and $48-58 \%$, respectively).[9, 14-16] Among patients with identifiable bleeding sources who received definite therapy by $\mathrm{DBE}$, there was a higher proportion of patients with ongoing OV than with previous OV (84\% versus $48 \%$ ). [9] It has been reported that patients with vascular lesions received the most benefit from an early DBE. [2,9] This may be because of the difficulty in demonstrating a small vascular lesion when the bleeding is inactive and/or when the procedure is delayed. Moreover, a previous study reported that vascular lesions were identified and received treatment either by endoscopy or surgery more frequently in the ongoing OV group (46\%), whereas only $19 \%$ of patients in the previous OV group and none in the OC bleeding group received prompt therapy [9]. Theoretically, all patients with OV who require urgent DBE should be classified as ongoing OV. In our series, the overall diagnostic yield in the urgent DBE group (70\%) was similar to that in the ongoing OV group from a previous study (77\%) [14]. We demonstrated that the urgent DBE group could identify more bleeding sources in OV than those in non-urgent DBE (70\% versus $30 \%, P<0.001$ ). Furthermore, the successful treatment rate was higher in patients who underwent urgent DBE than those who underwent non-urgent DBE ( $54 \%$ versus $15 \%, P<0.001$ ). In our study, we further subclassified the group of OV patients as "recent OV." Since urgent DBE can provide almost equally high diagnostic yields in ongoing and recent $\mathrm{OV}(75 \%$ versus $68 \%$, respectively, $P=0.67$ ), we therefore strongly suggest that urgent DBE should be performed not only in the ongoing OV group but also in the recent OV group.

Nevertheless, there is no consensus on how early enteroscopy should be performed in patients who present with OV. Only a small study, of only 10 patients, evaluating the benefit of emergency DBE (within $24 \mathrm{~h}$ ), showed a $90 \%$ diagnostic rate and all identifiable lesions received endoscopic therapy. [2] Based on the results of an earlier urgent capsule endoscopy study $(24-48 \mathrm{~h})$ in OV, it was reported that the diagnostic yield in 55 patients with OV was high (75\%). [3] However, there were limited numbers of patients with $\mathrm{OV}$ in those series and there were no direct comparisons between early and late capsule endoscopies. $[2,3,17]$ To be relevant to usual clinical practice, we defined the time of DBE by counting the time from the last episode of visible bleeding to the time when we performed DBE and used the term "urgent" DBE when it was performed within $72 \mathrm{~h}$ and those performed earlier than $72 \mathrm{~h}$ as "non-urgent" DBE. In practice, before OV could be diagnosed, upper and lower gastrointestinal bleeding sources have to be excluded $[1,5]$. Since some patients needed to undergo repeat standard endoscopy, or additional investigations including capsule endoscopy, angiography and nuclear scans $[1,5]$, we thought that $72 \mathrm{~h}$ would be appropriate to complete those investigations before commencing DBE. A recent series by Lecleire et al. reported that urgent capsule endoscopy (within 24-48 h) in patients with OV bleeding could identify possible bleeding lesions in $65 \%$ of patients [3]. However, 78\% of patients in the capsule series, with identifiable lesions, needed further therapeutic procedures, either endoscopy or surgery [3]. Although capsule endoscopy is a non-invasive diagnostic procedure permitting examination of the entire small bowel, it cannot provide endoscopic therapy or histological information. Moreover, capsule endoscopy is a timeconsuming procedure requiring at least $8-12 \mathrm{~h}$. A recent review 
recommended performing an early investigation in OV since there are greater chances of detecting bleeding sources and thus treatment [18]. However, choosing the most effective investigation is very challenging for this time constrained situation. Thus, our strategy to perform DBE first without prior capsule endoscopy in ongoing and recent OV might make more sense since diagnoses with potential therapeutic endoscopy can be made sooner [19]. The American Society for Gastrointestinal Endoscopy (ASGE) recommended angiography, capsule endoscopy, and DBE or a combination as diagnostic options for patients with OV [1]. The ASGE stated that initial DBE without prior capsule endoscopy would increase the endoscopy workload and endoscopic complications [1]. Therefore, they suggested an initial capsule endoscopy for almost all OVs. However, the guidelines [1] also stated DBE as first choice in certain patients and that endoscopy with therapeutic capability including DBE should be performed first in massive OV. Nevertheless, this leaves some unresolved issues, namely, the limitations of available technologies and the expertise of the endoscopist at each institution [18]. In our opinion, DBE, where available, should also be added as one of the first line approaches in ongoing and recent OV since we have shown that urgent DBE significantly increases therapeutic yield in these patients. Similar to the ASGE guidelines [1], we still recommend capsule endoscopy first for previous OV and inactive bleeding (previous OV) since there is a lower chance of identifying a causative lesion by DBE.

In our series, we demonstrated that an urgent DBE performed in patients with OV bleeding not only provided high diagnostic and therapeutic yields but also led to a lower rate of recurrent bleeding in patients whose bleeding sources were identified. Because of the small number of patients in our study, the lower recurrent bleeding rate (10\%) did not reach statistical significance. In our experience, the rebleeding rate was highest in patients with ulcers (44\%) and vascular lesions (40\%). This result is in agreement with the result from a recent study on long-term outcome after DBE (40\%) [13]. Hypothetically, these ulcers tend to be multiple and have different phases of ulcer healing. In addition, the original causes of the ulcer may not be completely removed or treated. These in turn could result in delayed or recurrent bleeding. For patients with vascular lesions, endoscopic treatment might have missed a certain small lesion that is a source of recurrent bleeding [12]. Moreover, many vascular lesions in our series were angiodysplasias ( Table 2 ) that tended to rebleed. On the other hand, no patient with a tumor developed recurrent bleeding, because those tumors were usually solitary, and all patients underwent complete resection. In addition, the possibility of another site for bleeding was rare [12].

There are several limitations in our study due to its retrospective nature. First, there was more blood transfused in the urgent DBE group than in the non-urgent group. However, when multivariate comparisons were analyzed, urgent DBE was the only independent factor for an increase in diagnostic yield whereas the amount of blood transfused before DBE (more than 4 units) was not significantly associated with the increase in diagnostic yield ( $64 \%$ versus $55 \%, P>0.05$ ). Second, there was a lower number of patients in the non-urgent than in the urgent DBE group. However, the result of our diagnostic yield in non-urgent bleeding (30\%) was in agreement with a study using capsule endoscope (13\%) [16] and a study that evaluated non-ongoing OV (47\%) (patients undergoing non-urgent DBE) [14]. Similar to other series, [14] ulcers and tumors were commonly detected in this type of bleeding. Second, $20 \%$ of our patients had incomplete follow-up data. However, the proportions of patients with complete follow-up data were equal in both groups ( $81 \%$ of the urgent DBE group and $80 \%$ of the non-urgent DBE group). Third, we did not perform the entire small bowel examination by DBE in all patients and some patients might have had more than one lesion. As far as the incomplete DBE examination was concerned, we concluded that all causative lesions had already been identified and treated. This in turn resulted in a low rebleeding rate comparable to other series $(<15 \%)$ [13]. Therefore, we did not think that a complete small bowel study was necessary, as long as the causative lesions were identified and treated appropriately. Lastly, none of our OV patients had capsule endoscopy prior to DBE. In our institution, the reimbursement for capsule endoscopy was very limited. In fact, we would not like to lose the golden study period $(8-12 \mathrm{~h})$ by adding capsule endoscopy to our protocol. Thus, our practice was to perform DBE in all patients with OV and we preferred to perform it as early as possible, and there were no complications related to DBE in our patients.

To date, there is no consensus on the optimal timing to perform DBE in patients with OV bleeding. This is the first study that demonstrated the advantage of performing DBE within $72 \mathrm{~h}$ after the last bleeding episode of OV. Nevertheless, before we can recommend an urgent DBE as the first-line approach to OV, especially in a patient with overt-ongoing bleeding, a further prospective study is warranted comparing urgent DBE versus urgent capsule endoscopy in different subgroups of OV, i.e., ongoing OV, recent $\mathrm{OV}$, and previous $\mathrm{OV}$.

In conclusion, in $\mathrm{OV}$, especially ongoing and recent $\mathrm{OV}$, urgent DBE (within $72 \mathrm{~h}$ ) provides higher diagnostic and therapeutic yields resulting in a lower rate of recurrent bleeding compared with non-urgent DBE.

\section{Competing interests: None}

\section{References}

1 Fisher L, Lee KrinskyM, Anderson MA et al. The role of endoscopy in the management of obscure GI bleeding. Gastrointest Endosc 2010; 72: $471-479$

2 Mönkemüller K, Neumann $H$, Meyer $F$ et al. A retrospective analysis of emergency double-balloon enteroscopy for small-bowel bleeding. Endoscopy 2009; 41: 715-717

3 Lecleire S, Iwanicki-Caron I, Di-Fiore A et al. Yield and impact of emergency capsule enteroscopy in severe obscure-overt gastrointestinal bleeding. Endoscopy 2012; 44: 337-342

4 Almeida N, Figueiredo P, Lopes $S$ et al. Urgent capsule endoscopy is useful in severe obscure-overt gastrointestinal bleeding. Dig Endosc 2009; 21: $87-92$

5 Raju GS, Gerson L, Das A et al. American Gastroenterological Association (AGA) Institute technical review on obscure gastrointestinal bleeding. Gastroenterology 2007; 133: 1697-1717

6 Nakamura M, Niwa $Y$, Ohmiya $N$ et al. Preliminary comparison of capsule endoscopy and double-balloon enteroscopy in patients with suspected small-bowel bleeding. Endoscopy 2006; 38: 59-66

7 Yamamoto H, Kita H, Sunada $K$ et al. Clinical outcomes of double-balloon endoscopy for the diagnosis and treatment of small-intestinal diseases. Clin Gastroenterol Hepatol 2004; 2: 1010-1016

8 Hadithi $M$, Heine GD, Jacobs MA et al. A prospective study comparing video capsule endoscopy with double-balloon enteroscopy in patients with obscure gastrointestinal bleeding. Am J Gastroenterol 2006; 101: 682

9 Tanaka S, Mitsui K, Yamada Y et al. Diagnostic yield of double-balloon endoscopy in patients with obscure GI bleeding. Gastrointest Endosc 2008; 68: 683-691

10 Estévez E, González-Conde B, Vázquez-Iglesias JL et al. Diagnostic yield and clinical outcomes after capsule endoscopy in 100 consecutive patients with obscure gastrointestinal bleeding. Eur J Gastroenterol Hepatol 2006; 18: 881-888 
11 Yano T, Yamamoto H, Sunada K et al. Endoscopic classification of vascular lesions of the small intestine (with videos). Gastrointest Endosc 2008; 67: 169-172

12 Shinozaki S, Yamamoto H, Yano T et al. Long-term outcome of patients with obscure gastrointestinal bleeding investigated by double-balloon endoscopy. Clin Gastroenterol Hepatol 2010; 8: 151-158

13 Fujita M, Manabe N, Honda K et al. Long-term outcome after doubleballoon endoscopy in patients with obscure gastrointestinal bleeding. Digestion 2010; 82: 173-178

14 Ohmiya N, Yano T, Yamamoto $H$ et al. Diagnosis and treatment of obscure GI bleeding at double balloon endoscopy. Gastrointest Endosc 2007; 66: $72-77$

15 Carey EJ, Leighton JA, Heigh RI et al. A single-center experience of 260 consecutive patients undergoing capsule endoscopy for obscure gastrointestinal bleeding. Am J Gastroenterol 2007; 102: 89-95
16 Pennazio $M$, Santucci R, Rondonotti $E$ et al. Outcome of patients with obscure gastrointestinal bleeding after capsule endoscopy: report of 100 consecutive cases. Gastroenterology 2004; 126: 643-653

17 Apostolopoulos P, Liatsos C, Gralnek IM et al. Evaluation of capsule endoscopy in active, mild-to-moderate, overt, obscure GI bleeding. Gastrointest Endosc 2007; 66: 1174-1181

18 Rondonotti E, Marmo R, Petracchini $M$ et al. The American Society for Gastrointestinal Endoscopy (ASGE) diagnostic algorithm for obscure gastrointestinal bleeding: eight burning questions from everyday clinical practice. Dig Liver Dis 2013; 45: 179-185

19 Gerson L, Kamal A. Cost-effectiveness analysis of management strategies for obscure GI bleeding. Gastrointest Endosc 2008; 68: 920-936 